\title{
In Situ Probe Approaches for Charge Reduction, Sample Manipulation, and Modified Total Release Lift-out
}

\author{
K. Langworthy ${ }^{1}$, J. Ditto ${ }^{1}$, C.D. Hartfield ${ }^{2}$, D. Krinsley ${ }^{3}$ \\ ${ }^{1}$ CAMCOR, University of Oregon, Eugene, OR 97403 \\ ${ }^{2}$ Omniprobe,Inc., Dallas, TX 75238 \\ ${ }^{3}$ University of Oregon, Dept. of Geology, Eugene, OR 97403
}

Since 1999 the FIB industry has been greatly changed by the development of commercial equipment designed for in situ lift-out (INLO). INLO uses a nano-manipulated probe to extract specimens from bulk material and has established itself as a dominant sample preparation method for TEM and atom probe microscopy. One particular method for INLO is the "total release" method, where samples are cut free from the bulk prior to attachment of the probe. [1] The total release method is generally accepted as a high-throughput method for INLO, and enables manipulation of in situ lift-out samples for plan-view preparation and sideways milling. [2,3] Our lab has creatively modified the total release method to generate a tab for probe attachment (further preserving regions of interest) that also allows the lamella to be stably suspended prior to probe attachment and lift out (thus ultimately increasing the success rate for attachment to the probe at desired angles).

Though INLO is a major use for in situ probes, we have found other novel uses particularly valuable to geological specimens and insulating samples. Traditional approaches for working with insulating samples typically involve the use of conductive coatings or use of variable pressure microscopes systems. [4] Coating samples can sometimes introduce unwanted artifacts, and access to variable pressure systems may be limited to some users. A new approach for local charge reduction employs the use of an in situ probe, placed very near the region of interest. Using the in situ probe for charge reduction allows users to image regions of insulating samples under high vacuum conditions without the use of coatings. We have also found the probe to be useful to manipulate and/or remove surfaces on geological specimens to expose features covered up by larger gains, sediment, or organic material.

\section{References:}

[1] T.M. Moore, U.S. Patent 6570170

[2] L. Zaykova-Feldman and T. M. Moore (2005). "Mechanical Conversion for High-Throughput TEM Sample Preparation." Microsc Microanal 11(2): 838-839

[3] C. Hartfield, M. Hammer et al. (2010). "Sample Repositioning Solutions for in situ Preparation and Analysis." Microsc Miroanal 16(S2): 16-17

[4] J. Goldstein et al. Scanning Electron Microscopy and X-ray Microanalysis, Kluwer Academic/Plenum Publishers, New York (2003). 


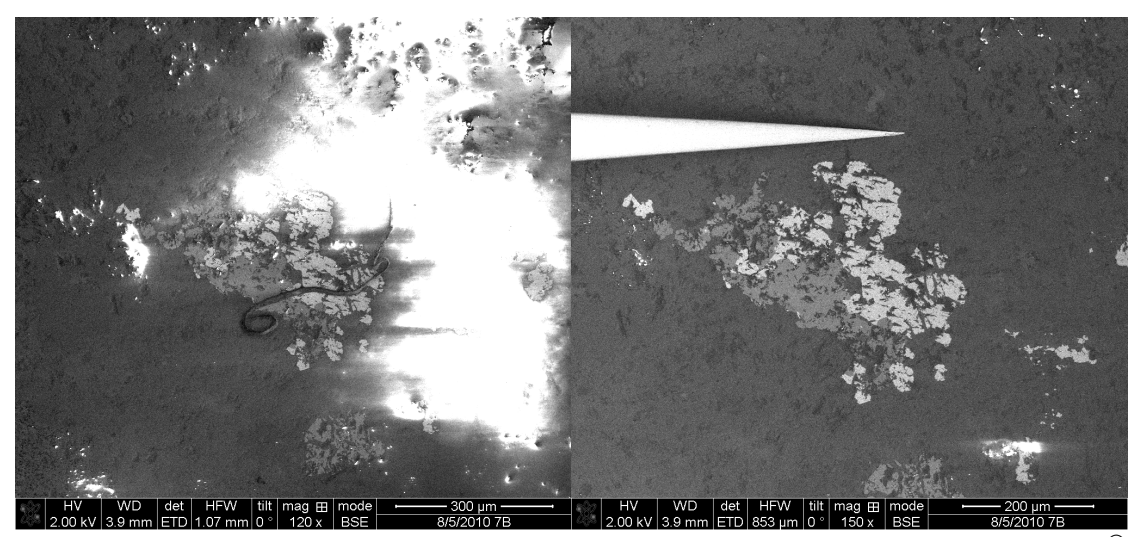

Figures 1 and 2: Uncoated, polished rock. (1) Without Autoprobe ${ }^{\circledR} 200$ nanomanipulator used for charge reduction. (2) With Autoprobe ${ }^{\circledR} 200$ nanomanipulator in contact with region of interest.

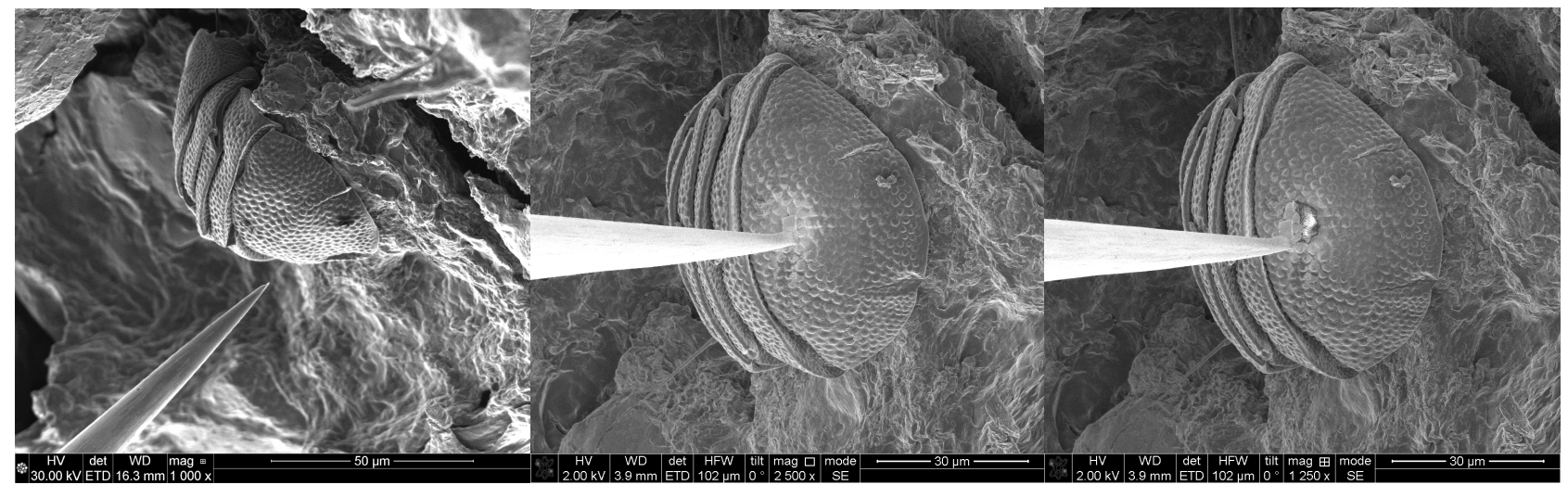

Figures 3,4,5: Manipulation/removal of surface from fossilized organism. (3) before attachment of probe, (4) during attachment using platinum gas, and (5) after removal of surface layer.
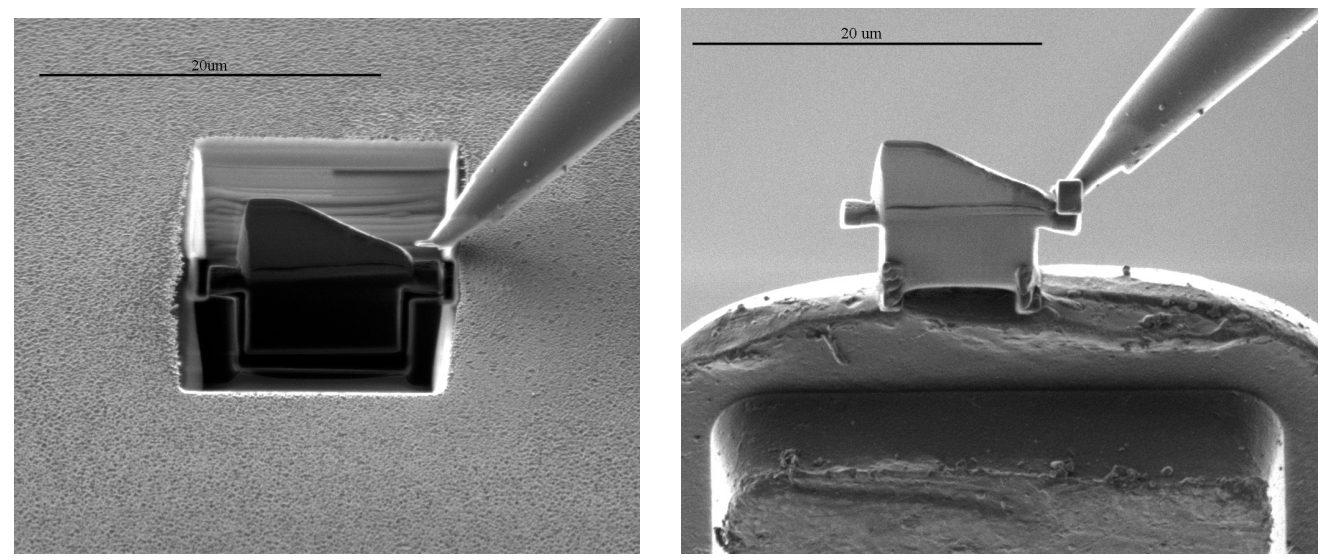

Figures 6 and 7: Modified total release method. (6) Freely suspended lamella in trench prior to probe attachment. (7) Attached to Autoprobe ${ }^{\circledR} 200$ nanomanipulator (by tab) while transferring to a TEM grid. 